Vol. 23, 2021/e12

\title{
Valoración de la calidad creativa de ideas contextualizando su proceso
}

\section{Assessing the Creativeness of Ideas in a Contextualized Process}

Estela de Lourdes Juárez Ruiz (1) https://orcid.org/0000-0002-2857-0772

Jorge Alfredo Lombardero Chartuni (2) https://orcid.org/0000-0001-7783-6664

Lidia Aurora Hernández Rebollar (1) https://orcid.org/0000-0003-0658-4396

(1) Benemérita Universidad Autónoma de Puebla

(2) Universidad de Guadalajara

(Recibido: 4 de abril de 2019; Aceptado para su publicación: 12 de agosto de 2019)

Cómo citar: Juárez, E. de L., Lombardero, J. A. y Hernández, L. A. (2021). Valoración de la calidad creativa de ideas contextualizando su proceso. Revista Electrónica de Investigación Educativa, 23, e12, 1-13. https://doi.ora/10.24320/redie.2021.23.e12.2964

\section{Resumen}

En el contexto de las investigaciones actualmente se sugiere utilizar esfuerzos deliberados para cultivar la creatividad, razón por la cual el presente estudio propone emplear en un proceso contextualizado, las técnicas la ficha de idea y la sesión de creatividad con una modificación en una de sus etapas, para promover la generación de ideas creativas. Se utilizó un diseño experimental con únicamente postest. En el grupo experimental los estudiantes propusieron el problema a resolver mientras que, en el grupo de control, los alumnos resolvieron un problema establecido por el facilitador. Los resultados muestran diferencias estadísticamente significativas en la escala de valoración de las ideas en las dimensiones novedad, originalidad y elaboración en el grupo experimental con respecto del grupo de control, lo cual muestra que la elección o búsqueda del problema a resolver puede promover la generación de ideas creativas.

Palabras clave: generación de ideas creativas, pensamiento creativo, planteamiento creativo de problemas, solución creativa de problemas

\section{Abstract}

The current research context recommends employing conscious efforts to cultivate creativity. This study proposes the use of idea sheets and creativity sessions (with a change in one stage), within a contextualized process, to promote the generation of creative ideas. Following a posttest-only experimental design, the students in the experimental group proposed the problem to be solved while the control group solved a problem provided by the facilitator. The results show statistically significant differences in the idea assessment scale in the dimensions novelty, originality, and elaboration in the experimental group with respect to the control group, showing that choosing or searching for a problem to be solved can promote the generation of creative ideas. 


\section{Introducción}

En la actualidad la creatividad ya no es opcional, se ha convertido en una habilidad crítica que debe ser incluida en los procesos de aprendizaje en instituciones educativas para preparar profesionales altamente competitivos (Wu et al., 2018). Los investigadores y profesionales se han preocupado por estudiar estrategias de aprendizaje para su desarrollo en individuos y grupos; particularmente se han enfocado en mejorar las herramientas y métodos utilizados para apoyar la producción de ideas (Richardson y Mishra, 2018; Tran et al., 2017), ya que la capacidad de generar o procesar ideas creativas es fundamental para promover la innovación (García et al., 2018) y fomentar las capacidades de sistematización del pensamiento creativo y su concreción a través de la solución de problemas contextualizados. La universidad busca insertarse en la sociedad como motor del desarrollo económico y social mediante la generación y el desarrollo de ideas creativas.

Sin embargo, se requiere seguir aprendiendo sobre las formas en que los docentes o facilitadores puedan crear entornos que apoyen la generación de ideas creativas en los aprendices (Liao et al., 2018). Es necesario experimentar con propuestas experienciales en el aula; una forma posible es estableciendo el vínculo entre el desarrollo de ideas creativas y los problemas reales del contexto, pues la creatividad es un proceso que sensibiliza ante los problemas y permite identificar dificultades y buscar soluciones (Torrance, 1972 como se citó en Ordaz et al., 2016).

El propósito de esta investigación es promover el desarrollo de la creatividad en estudiantes o aprendices, a través de una propuesta de proceso contextualizado que pueda ser implementado por facilitadores de cualquier disciplina. Asimismo, identificar, durante su implementación, posibles diferencias en la calidad creativa de las ideas desarrolladas por los estudiantes, en dos alternativas: 1) los aprendices proponen el problema a resolver o 2) atienden un problema del contexto establecido previamente por el facilitador.

Por ello, la pregunta de investigación fue: ¿Qué diferencias significativas se identifican en la valoración de la calidad creativa de ideas producidas cuando un problema es propuesto por aprendices o cuando es provisto por el facilitador, en un proceso creativo contextualizado? De ésta se deriva el objetivo: Identificar diferencias significativas en la valoración de la calidad creativa de ideas producidas cuando un problema es propuesto por aprendices o cuando es provisto por el facilitador, en un proceso creativo contextualizado.

Atender este problema puede coadyuvar a diseñar mejores ecosistemas creativos en las aulas estudiantiles, repensando el papel del facilitador en ambos contextos. Para cumplir con el objetivo se plantea la hipótesis: la calidad de las ideas producidas por los estudiantes que identifican el problema a resolver es significativamente mayor de los estudiantes que atienden un problema establecido por el facilitador.

\subsection{Propuesta de proceso contextualizado para la generación de ideas creativas}

La creatividad es el mejor ejemplo de la naturaleza dinámica de la inteligencia, y puede requerir todas las áreas de nuestra mente y de nuestro ser (Robinson y Aronica, 2014). Involucra procesos de exploración, imaginación y pensamiento creativo que ocurren con base en el conocimiento, la motivación, las emociones y las experiencias (Tran et al., 2017). Emerge de la interacción entre ciertas actitudes, procesos cognitivos específicos y el medio ambiente en el cual el individuo o los grupos se encuentran (Plucker et al., 2004).

Pueden ser utilizados esfuerzos deliberados para cultivar aptitudes creativas, así como procesos para mejorar la probabilidad para la creatividad (Perry y Karpova, 2017; Plucker et al., 2004; Vally et al., 2019).

Por esto, se considera en primer lugar, el estudio y análisis de la creatividad. Inicialmente se establece la imaginación como la capacidad de crear imágenes. Que es una función psíquica, compleja, dinámica, estructural, que puede provocarse por motivaciones de diverso orden: perceptual, racional, instintiva, 
afectiva, etc. Que puede ser consciente o inconsciente; subjetiva u objetiva, de orden externo al sujeto. Que actúa en diversos niveles y contextos y participa en recuerdos, conceptos, lenguaje, ensueños, en la creación artística en las creencias colectivas y la investigación científica y tecnológica (Lapoujade, 1988); y puede formar la capacidad de pensar creativamente (Wu et al., 2018).

En el análisis de la imaginación se propone hacer énfasis en la importancia del lenguaje, específicamente de las palabras como contenedoras de ideas, y en la capacidad de estar en flujo (Csikszentmihalyi, 2014); es decir, la sensación que las personas reportan cuando las habilidades se vuelven su segunda naturaleza en donde todo lo que se hace parece venir naturalmente, y cuando la concentración es tan intensa que se pierde la noción del tiempo. Csikszentmihalyi argumenta que es esta sensación óptima de flujo la que alimenta el motor de la motivación intrínseca que impulsa la creatividad; es decir, la experiencia óptima.

El principio de motivación intrínseca es un estado en el que las personas son más creativas, se sienten motivadas principalmente por el interés, el disfrute, la satisfacción y el desafío del trabajo en sí (Amabile, 2013); es el deseo de sentirse competente y autodeterminado (Bretschneider et al., 2012).

Por otro lado, para el concepto de creatividad se plantea trabajar con la propuesta de Galvao et al. (2009), quienes la consideran un fenómeno complejo, multidimensional, plurideterminado y dinámico. Que se revela a partir de procesos mentales tales como el lenguaje, la toma de decisiones y la memoria. Que considera cuatro aspectos llamados las cuatro P's por sus siglas en inglés: Person, las características de la persona; Process, el proceso de creación; Product, los elementos presentes en el producto creativo, y Press (por ejemplo, environment), los factores del ambiente donde el individuo se encuentra inmerso (Rhodes, 1962, como se citó en Baer y Kaufman, 2019). En el estudio de este tema se propone también analizar las cualidades que promueven la creatividad (Robinson y Aronica, 2014).

Se establece también el estudio de algunas técnicas de creatividad. En específico: la sesión de creatividad, el warm-up (calentamiento), la lluvia de ideas, la matriz de descubrimiento, la ficha de idea, el estudio creativo (Benoit, 2011), y las cuatro gorras de la creatividad (Gold, 2007).

Por otro lado, dado que la creatividad también se asocia con la capacidad de formulación y resolución de problemas (Elisondo, 2015), algunos programas de capacitación incluyen la búsqueda y solución creativa de problemas (Amabile, 1996; Byrge y Tang, 2015; Gruner y Csikszentmihalyi, 2019). Estos elementos se han identificado como un recurso que activa procesos cognitivos divergentes, flexibles y alternativos y permite examinar la creatividad de los estudiantes al analizar la novedad de las soluciones (Вarrera-Mora y Reyes-Rodríguez, 2017; Richardson y Mishra, 2018).

Por lo anterior, en segundo lugar se propone la actividad de planteamiento de problemas contextualizados. Además, se requiere considerar un ambiente propicio para fomentar la creatividad. Richardson y Mishra (2018) proponen ambientes de aprendizaje basados en la pasión, la co-creación y la colaboración, y una atmósfera en la cual las ideas son valoradas y los errores vistos como una parte necesaria del proceso de aprendizaje. Asimismo, Newton (2013) indica que los estados de ánimo y las emociones también pueden influir en la creatividad en la escuela y afirma que la solución de problemas en equipos colaborativos puede coadyuvar a un estado de ánimo positivo, ya que fomenta la cooperación.

Tomando en cuenta lo anterior, se propone establecer un espacio de aprendizaje que considere la reflexión compartida, el análisis de conceptos y el juego, a través de actividades flexibles diseñadas para este fin. En específico, se propone llevar a cabo en el aula la técnica creativa sesión de creatividad (Benoit, 2011) (ver Figura 1) con una modificación en el cuarto paso, como se describe a continuación. 
Figura 1. La sesión de creatividad

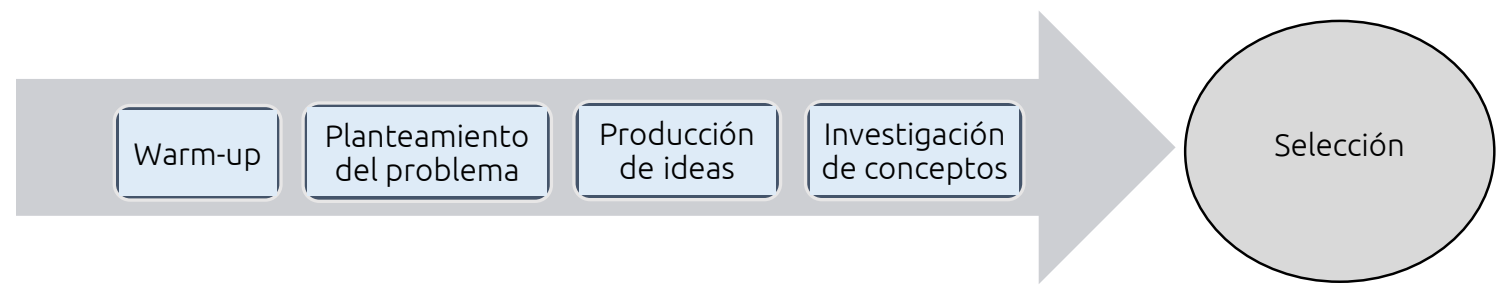

Fuente: Benoit (2011).

Inicialmente se realiza el warm-up en plenaria, realizando algunos juegos como anagramas y progresiones de palabras. Esta actividad motiva al aprendiz y despierta la creatividad para la solución de problemas complejos (Zatarain, 2018), relaja su mente, permite que fluyan las ideas y ayuda a trabajar también con las ideas de los demás. A continuación, en equipos colaborativos se propone realizar la actividad del planteamiento de un problema del contexto y la actividad producción de ideas (pensamiento divergente) aplicando la técnica lluvia de ideas. Después, en sustitución de la investigación de conceptos (ver Figura 1) se aplica la técnica creativa "ficha de idea" para que los estudiantes puedan enfocarse en desarrollar y describir su idea. Esta misma técnica permite a los participantes decidir si su idea está madura, si tendrán que seguir trabajando en ella o si es rechazada.

En la parte del planteamiento del problema (Figura 1) es donde se estableció un marco de diferenciación para la prueba experimental de la hipótesis. Al grupo experimental se le dio la libertad de plantear el problema a resolver, mientras que al grupo de control se le proporcionó la situación problema descrita en la Figura 2, la cual fue diseñada por los facilitadores.

Figura 2. Situación problema del puente peatonal

La Benemérita Universidad Autónoma de Puebla en Ciudad Universitaria posee accesos de entrada-salida alrededor de su periferia. Algunos de ellos resultan peligrosos para la comunidad. Por ello, las autoridades gubernamentales tomaron diversas medidas de seguridad, no sólo para los universitarios sino para cualquier peatón, entre otras, la construcción de puentes peatonales y accesos controlados. Pese a ello los accidentes en cuanto al cruce de vialidades son cada vez más frecuentes, sobre todo del lado del Boulevard Valsequillo.

El puente peatonal más cercano a la Facultad de Arquitectura se construyó hace poco más de ocho años, tiene un diseño basado en rampas con diversas inclinaciones cuya finalidad es la de facilitar el cruce peatonal de personas, sin embargo, se ha detectado que casi no se usa a pesar de los riesgos y peligros que presenta esta vialidad. Gran parte de esta situación se debe a las razones manifestadas por algunos usuarios como: tiene difícil acceso, sus rampas son muy pronunciadas, es resbaloso en temporada de lluvias, pierden tiempo, les da flojera, está muy alto, no es fácil de usar por personas discapacitadas o de edad avanzada, invade el libre tránsito de los peatones por la acera, asaltan, los cables de luz y teléfono se hallan junto al barandal.

Con respecto a la técnica creativa "ficha de idea", se trata de un documento escrito que es elaborado por los participantes, el cual está conformado por los siguientes elementos: título de la idea, descripción, dibujo o esquema, ventajas, inconvenientes, variantes, métodos de realización, problemas planteados, respuesta y conclusión (Benoit, 2011). En nuestro estudio, los estudiantes realizaron su ficha de idea en una presentación en Power Point en equipos colaborativos.

Por último, para valorar la calidad creativa de las ideas desarrolladas por los estudiantes a través de su ficha de idea, se considera la escala de calidad creativa de las ideas propuesta por Bretschneider et al. (2012), la cual está conformada por siete dimensiones: Novedad, Originalidad, Paradigma relacionado, Factibilidad, Aceptabilidad, Efectividad y Elaboración (Tabla 1). 
Tabla 1. Dimensiones de calidad de una idea creativa

\begin{tabular}{|c|c|}
\hline Dimensión & Definición \\
\hline Novedad & Grado en que la idea es rara y nadie la ha expresado antes en ese contexto. \\
\hline Originalidad & Grado en que la idea es inusual, visionaria y sorprendente. \\
\hline Paradigma relacionado & $\begin{array}{l}\text { Grado en que una idea ayuda a superar las estructuras establecidas, es decir, } \\
\text { cuán radical o revolucionaria es. }\end{array}$ \\
\hline Factibilidad & Grado en que una idea puede ser implementada. \\
\hline Aceptabilidad & Grado en que una idea es aceptada social, legal o políticamente por otros. \\
\hline Efectividad & Grado en que una idea resolverá un problema. \\
\hline Elaboración & Grado en que una idea es completa, detallada y comprensible. \\
\hline
\end{tabular}

Fuente: Bretschneider et al. (2012).

Traducción propia.

\section{Método}

Se trató de un diseño experimental con dos grupos, con postest únicamente, que Campbell y Stanley (1973, p. 54) señalan es pertinente en los casos en los que se tiene que experimentar "con métodos que permiten la introducción inicial de elementos absolutamente nuevos, para los cuales son imposibles los pretests en el sentido ordinario del término". En la investigación que se reporta este es el caso, ya que los estudiantes sujetos de la investigación no tenían conocimientos previos acerca de los temas del taller ni del instrumento utilizado para medir el nivel creativo de las ideas.

El diseño del experimento para la recolección de datos consistió, inicialmente, en reunir a la población de sujetos participantes en un solo grupo, y durante todas las sesiones (excepto en la última) les fue impartido el taller de creatividad por dos facilitadores, que fueron los mismos que hicieron el diseño del taller. En la última sesión se seleccionó el grupo de control y el grupo experimental a través de un muestreo aleatorio por conglomerado, colocándolos en dos salones con computadoras. Se designó aleatoriamente cuál de ellos sería el grupo de control y cuál el experimental y se determinó también de manera aleatoria qué facilitador estaría en cada grupo. En el grupo de control el facilitador proporcionó el problema contextualizado a trabajar (descrito en la Figura 2), mientras que en el grupo experimental los estudiantes propusieron el problema a resolver.

Рara la prueba de hipótesis la variable independiente se consideró el problema contextualizado con dos valores: el problema es proporcionado por el facilitador, y el problema es planteado por los estudiantes. La variable dependiente o variable de salida fue la calidad creativa de las ideas producidas por cada grupo.

La población de estudio consistió en los estudiantes de nuevo ingreso de la Universidad Politécnica Metropolitana de Puebla, en México, inscritos en el primer semestre en el período comprendido entre agosto y diciembre de 2018. La selección del grupo de control y del grupo experimental se realizó a través de un muestreo aleatorio por conglomerado.

El instrumento utilizado para la recolección de datos consistió en la ficha de idea (Benoit, 2011). Para valorar la calidad creativa de las ideas se utilizó la escala de Bretschneider et al. (2012), con un rango de calificación de 1 (el más bajo) a 4 (el más alto). La escala presentó una validez de constructo aceptable (Amabile, 1996, como se citó en Bretschneider et al., 2012), con coeficientes de correlación de clase entre 0.571 y 0.834 .

\section{Resultados}

De los 64 estudiantes participantes 29 fueron mujeres (45.3\%) y 35 hombres (54.7\%), con edades entre 18 y 21 años con una media de 18.7. El taller se realizó del 16 de octubre al 6 de noviembre de 2018 en cuatro sesiones semanales de dos horas. Los estudiantes estuvieron distribuidos en las cuatro licenciaturas que ofrece la universidad (ver la Tabla 2). La ingeniería en Biotecnología tuvo la mayor cantidad de mujeres (16) y la ingeniería en Sistemas computacionales mayor cantidad de hombres (17). 
Tabla 2. Distribución de la muestra

\begin{tabular}{lccc}
\hline Licenciatura & $n$ & Hombres & Mujeres \\
\hline Ingeniería en Biotecnología & 22 & 6 & 16 \\
Ingeniería en Logística y Transportes & 9 & 4 & 5 \\
Ingeniería en Sistemas Computacionales & 19 & 17 & 2 \\
Licenciatura en PYMES & 14 & 8 & 6 \\
\hline Total & 64 & 35 & 29 \\
\hline
\end{tabular}

El grupo de control contó con 36 estudiantes distribuidos en 13 equipos de 2, 3 o 4 participantes, mientras que el grupo experimental tuvo 28 estudiantes distribuidos en 11 equipos de 2, 3 o 4 participantes. Cada equipo realizó una ficha de idea, y cada una de ellas fue evaluada por los facilitadores, los cuales discutieron cada una en conjunto.

Un ejemplo de problema planteado por el grupo experimental fue el de los desechos orgánicos sólidos de los perros domésticos. La idea que los estudiantes propusieron para resolver este problema la presentaron en su ficha de idea, de la cual se muestran algunas diapositivas en la Figura 3. En su ficha afirmaron:

El objetivo principal del proyecto a futuro es lograr la creación de combustible y abono, dándole utilidad a estos desechos, y para poder lograrlo se necesitaría cambiar la alimentación del perro con el fin de que el producto sea orgánico y no sea dañino tanto al medio ambiente como a los seres humanos ya que la sustancia que sueltan las heces es ácido sulfhídrico generado de la digestión anaerobia que al mezclarse con otros desechos orgánicos o agua pueden provocar ácidos corrosivos por lo que deben tomarse precauciones.

En su descripción anotaron: "Elaboración de un alimento ecológico nutritivo para perros domésticos con ayuda de residuos orgánicos con el objetivo de que tenga una mejor alimentación para así poder aprovechar sus heces fecales y llegar a producir gas".

Llama la atención de su idea que en la diapositiva Problemas planteados hicieron la propuesta de convertir a los perros en veganos.

Figura 3. Algunos elementos de la ficha "Rogin Can: Alimento ecológico"
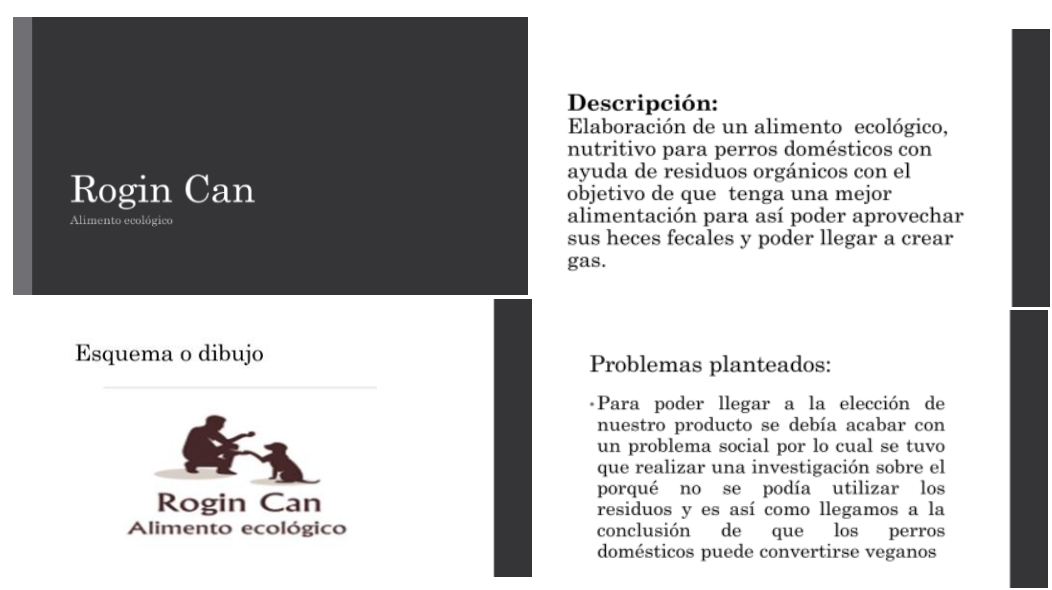

Esta idea fue evaluada en el más alto nivel en las dimensiones: novedad, originalidad, paradigma relacionado y elaboración (ver Figura 4), ya que incluso describieron en una receta cómo elaborar las croquetas para los perros (la diapositiva no se incluye por respeto a la originalidad de su idea). 
Figura 4. Evaluación de la idea "Rogin Can: Alimento ecológico"

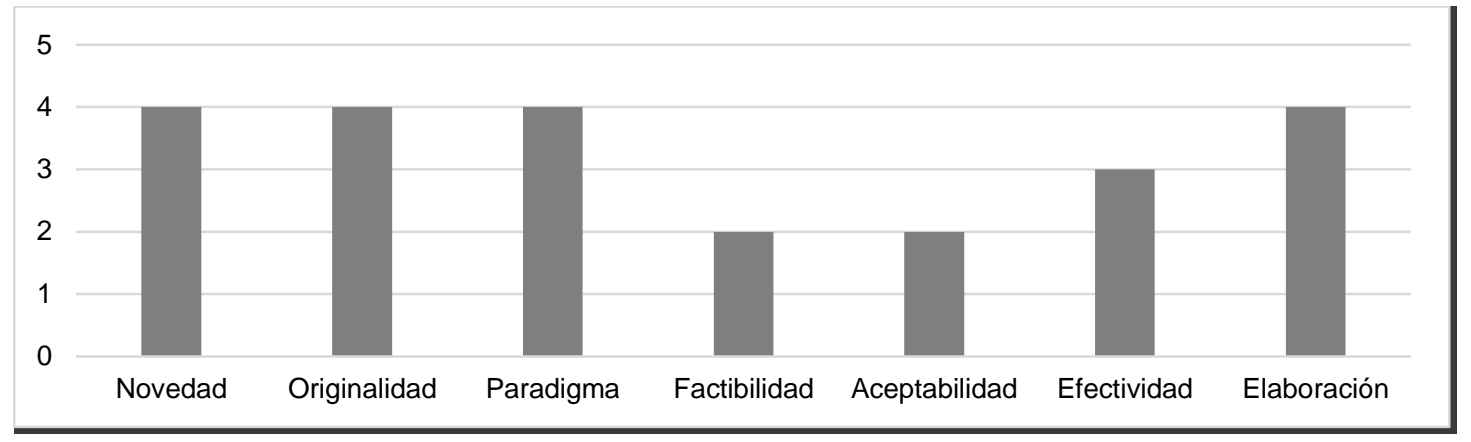

Por su parte, un ejemplo del grupo de control fue la idea "Empezando a reconstruir Puebla", que atendió el problema del puente peatonal, la cual fue planteada en su ficha de idea con algunos elementos que se muestran en la Figura 5.

En su descripción mencionaron: "Somos jóvenes que se preocupan por la seguridad de sus compatriotas. Vamos a cambiar todo aquello que no está seguro para los ciudadanos para su comodidad (...) No solamente debemos hablar, sino hablar, actuar y poner en acción todo aquello que vemos mal. Oración y acción. Muévete, mídete, quiérete." Como se puede observar, en estas oraciones no describieron cuál era su idea para solucionar el problema. En su esquema o dibujo incluyeron la imagen de un puente con un diseño muy moderno que se interpreta es la idea que ellos propusieron, ya que no la explicaron con palabras, la cual, como puede observarse no es una idea original.

En los medios de realización establecieron: "Conversando con gente de dinero y haciéndolos recapacitar para que nos den el apoyo económico", lo que en la evaluación de la ficha de idea permitió evaluar la dimensión "Factibilidad".

Figura 5. Elementos de la ficha de idea: Empezando a reconstruir Puebla

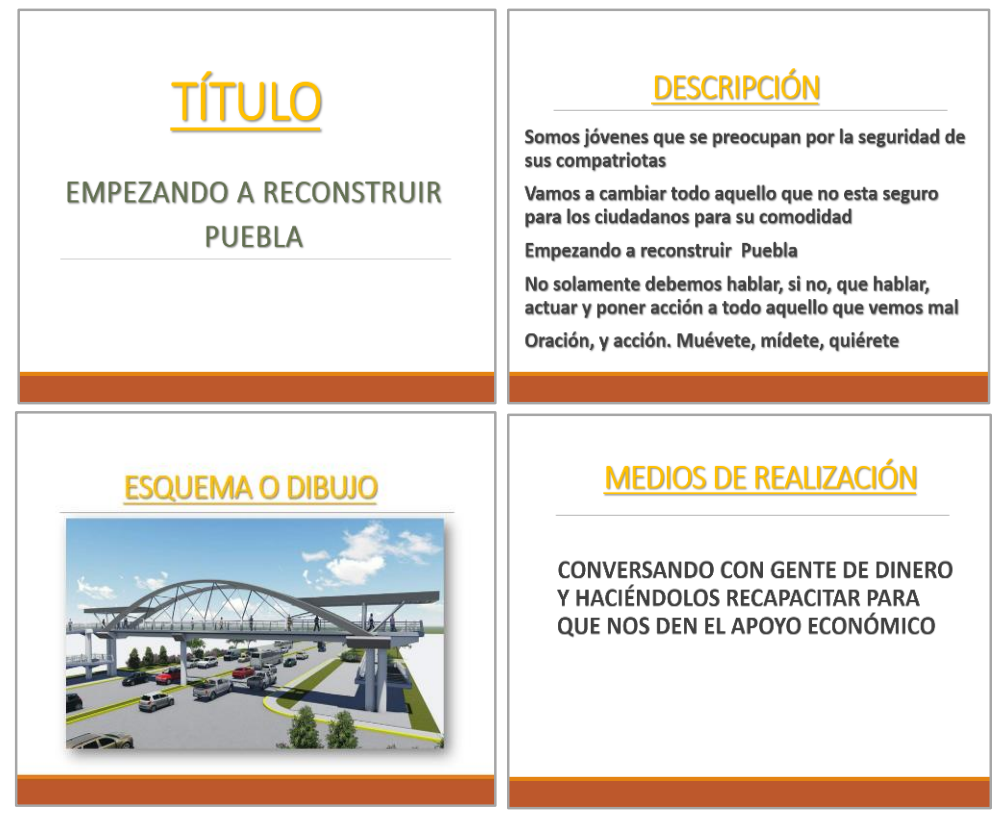

En la Figura 6 se muestra la valoración de las siete dimensiones de calidad creativa de esta idea, en la que se apreciar que sólo alcanzaron el Nivel 3 en la dimensión aceptabilidad, mientras que en novedad, efectividad y elaboración obtuvieron la calificación más baja (Nivel 1). 
Figura 6. Evaluación de la idea: Empezando a reconstruir Puebla

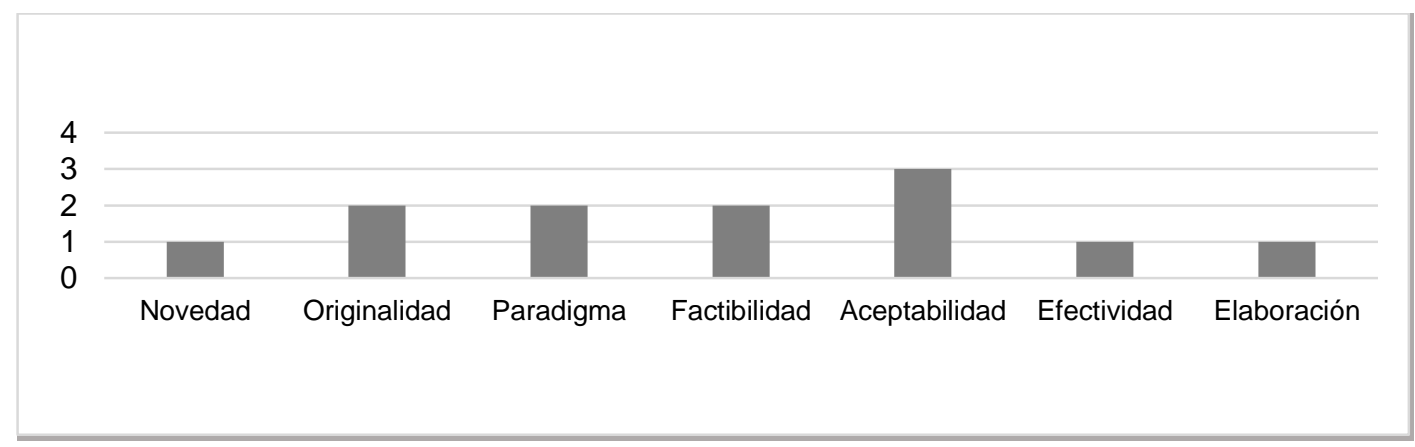

El análisis no paramétrico a través de las pruebas U de Mann-Whitney y Wilcoxon-Mann-Whitney tuvo como objetivo identificar posibles diferencias significativas entre el grupo de control y el grupo experimental en cada una de las dimensiones de calidad creativa de las ideas: novedad, originalidad, paradigma relacionado, factibilidad, aceptabilidad, efectividad y elaboración. Para ello, se planteó la siguiente prueba estadística:

$$
\begin{aligned}
& \text { Ho: } P(X>Y)=P(Y>X) \\
& \text { Ha: } P(X>Y) \neq P(Y>X)
\end{aligned}
$$

Donde $X$ e $Y$ denotan observaciones del grupo de control y grupo experimental, respectivamente.

Los datos presentaron alguna evidencia de no normalidad a través de las pruebas Shapiro-Wilk, Kolmogorov-Smirnov y Jarque Bera, como se puede observar en la Tabla 3.

Tabla 3. Pruebas de normalidad de los datos para cada dimensión

\begin{tabular}{lcccccc}
\hline \multirow{2}{*}{ Dimensión } & \multicolumn{2}{c}{ Shapiro-Wilk } & \multicolumn{2}{c}{ Kolmogorov-Smirnov } & \multicolumn{2}{c}{ Jarque Bera } \\
\cline { 2 - 7 } & $\mathrm{W}$ & $\mathrm{P}$ & $\mathrm{D}$ & $\mathrm{p}$ & $\mathrm{x}^{2}$ & $\mathrm{P}$ \\
\hline Grupo de Control & & & & & & \\
\hline Novedad & 0.807 & 0.011 & 0.421 & 0.028 & 0.854 & 0.653 \\
Originalidad & 0.778 & 0.005 & 0.432 & 0.023 & 2.7259 & 0.256 \\
Paradigma & 0.802 & 0.009 & 0.299 & 0.233 & 1.132 & 0.568 \\
Factibilidad & 0.699 & 0.001 & 0.392 & 0.050 & 0.505 & 0.777 \\
Aceptabilidad & 0.777 & 0.005 & 0.319 & 0.173 & 1.245 & 0.537 \\
Efectividad & 0.895 & 0.137 & 0.227 & 0.569 & 0.495 & 0.781 \\
Elaboración & 0.807 & 0.011 & 0.257 & 0.407 & 0.854 & 0.653 \\
\hline Grupo experimental & & & & & \\
\hline Novedad & 0.780 & 0.005 & 0.385 & 0.077 & 1.395 & 0.498 \\
Originalidad & 0.814 & 0.014 & 0.372 & 0.095 & 1.221 & 0.543 \\
Paradigma & 0.863 & 0.064 & 0.249 & 0.502 & 0.967 & 0.616 \\
Factibilidad & 0.795 & 0.008 & 0.294 & 0.298 & 1.210 & 0.547 \\
Aceptabilidad & 0.785 & 0.006 & 0.274 & 0.382 & 1.22 & 0.545 \\
Efectividad & 0.877 & 0.095 & 0.334 & 0.172 & 0.66 & 0.719 \\
Elaboración & 0.833 & 0.025 & 0.227 & 0.621 & 0.624 & 0.732 \\
\hline
\end{tabular}

Las pruebas de hipótesis tuvieron como resultado un nivel de significancia ( $p$-valor $<0.05$ ) en tres dimensiones: novedad, originalidad y elaboración, por lo tanto, se rechaza la hipótesis nula y se afirma que la probabilidad de que las observaciones de una población superen a las de la otra difiere en forma significativa (Tabla 4). 
Tabla 4. Resumen de las pruebas de hipótesis estadísticamente significativas

\begin{tabular}{lccccc}
\hline & \multicolumn{2}{c}{ U de Mann-Whitney } & \multicolumn{2}{c}{ Wilcoxon-Mann Whitney } \\
\cline { 2 - 5 } Dimensión & W & $p$ & Z & $p$ & TE \\
\hline Novedad & 33.5 & 0.03990 & -1.9820 & 0.0485 & -0.4321 \\
Originalidad & 31.5 & 0.02931 & -2.2114 & 0.0262 & -0.4611 \\
Elaboración & 35 & 0.04472 & -2.0402 & 0.0446 & -0.4254 \\
\hline
\end{tabular}

Nota: TE = tamaño del efecto.

Mediante las pruebas U de Mann-Whitney y Wilcoxon-Mann-Whitney se identificó una diferencia estadísticamente significativa entre el grupo de control y el grupo experimental en la novedad de la idea, ya que las pruebas generaron un nivel de significancia con un $p$-valor de 0.039 y 0.0385 , respectivamente (ver Tabla 4). En los diagramas mostrados en la Figura 7 se puede observar que la mediana del grupo de control (A) está en el nivel 2 (no tan común), con un valor máximo de 3 (un poco rara), mientras que en el grupo experimental (B) la mediana se encuentra en el nivel 3 mostrando que el grupo experimental tuvo una calificación significativamente mayor en la novedad de la idea que el grupo de control.

Figura 7. Novedad de la idea del grupo de control (A) y del grupo experimental (B)

\section{Novedad grupo A Novedad grupo B}
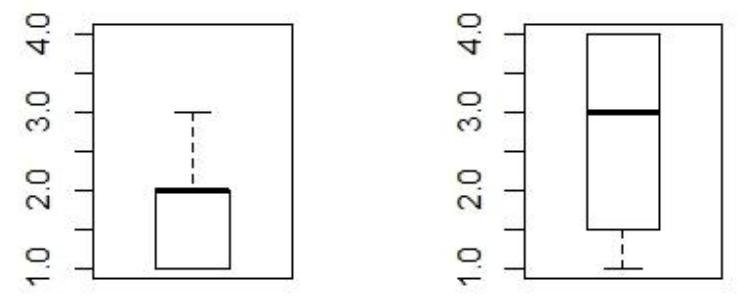

Nota: Escala: 1 = común, 2 = no tan común, 3 = un poco rara, 4 = rara, inusual.

Asimismo, a través de las pruebas $U$ de Mann-Whitney y Wilcoxon-Mann-Whitney se identificó una diferencia estadísticamente significativa entre el grupo de control y el grupo experimental en la originalidad de la idea, pues las pruebas de hipótesis generaron un nivel de significancia con un $p$-valor de 0.029 y 0.026 , respectivamente. En los diagramas de cajas y bigotes mostrados en la Figura 8 se puede observar que el grupo de control tiene una mediana de 1.5 (promedio entre aburrida y algo interesante) con un valor máximo de 3 (más interesante), mientras que el grupo experimental alcanzó una mediana en el nivel 3 con un tercero y cuarto cuartil en 4 (sorprendente), lo que marcó una diferencia estadísticamente significativa.

Figura 8. Originalidad de la idea del grupo de control (A) y del grupo experimental (B)

\section{Originalidad grupo A Originalidad grupo B}
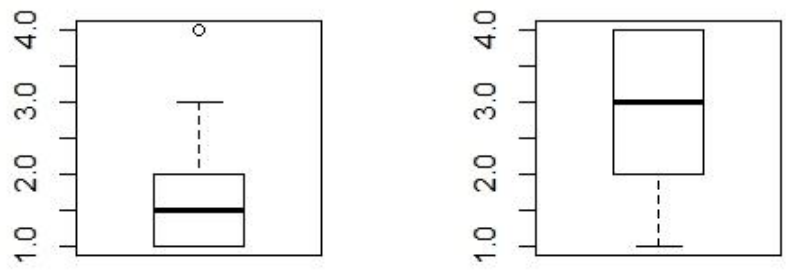

Nota: Escala: 1 = aburrida, 2 = algo interesante, 3 = más interesante, 4 = sorprendente. 
Finalmente, mediante las pruebas U de Mann-Whitney y Wilcoxon-Mann-Whitney se identificó la posible diferencia estadísticamente significativa entre el grupo de control y el grupo experimental en la elaboración de la idea, ya que la hipótesis a través de ambas pruebas generó un nivel de significancia con un $p$-valor de 0.0447 y 0.0446, respectivamente. En la Figura 9 se puede observar que el grupo de control obtuvo una mediana de 2 (comprensible) y un máximo valor de 3 (detallada), mientras que el grupo experimental tuvo una mediana de 3 (detallada), un tercer cuartil en 3.5 y máximo en 4 (completa).

Figura 9. Elaboración de la idea del grupo de control (A) y del grupo experimental (B)

\section{Elaboración grupo A Elaboración grupo B}
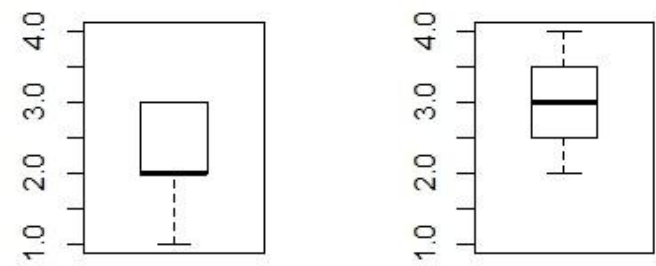

Nota: Escala: 1 = poco comprensible, 2 = comprensible, 3 = detallada, 4 = completa.

\section{Discusión y conclusiones}

Se realizó una propuesta para promover la generación de ideas creativas en estudiantes de licenciatura, a través de un proceso contextualizado que utilizó dos técnicas de creatividad: la ficha de idea y la sesión de creatividad, teniendo esta última una propuesta de modificación en una de sus etapas. La propuesta se implementó en estudiantes de nuevo ingreso a la universidad. El diseño experimental fue de dos grupos con postest únicamente. En el grupo de control el facilitador estableció el problema contextualizado a trabajar, mientras que en el grupo experimental los estudiantes propusieron el problema. Los hallazgos del estudio fueron que los participantes del grupo experimental desarrollaron ideas de mayor calidad creativa que los estudiantes del grupo de control.

Las dimensiones en la escala de calidad creativa de las ideas propuestas por Bretschneider et al. (2012) mostraron diferencias estadísticamente significativas entre el grupo de control y el experimental en la novedad, originalidad y elaboración de las ideas, en concordancia con el estudio de Byrge y Tang (2015), quienes también obtuvieron mejoras significativas en su estudio de entrenamiento creativo en las dimensiones autoeficacia creativa, originalidad, elaboración y fluidez.

El factor de diferenciación en el experimento de plantear o no el problema a resolver, marcó una diferencia estadísticamente significativa en la calidad creativa de las ideas propuestas, en concordancia con Newton (2013), quien estableció que la búsqueda del problema (en lugar de trabajar con un problema provisto por el maestro) y su solución, pueden generar experiencias emocionales intensas, posiblemente debido a la cantidad de inversión personal.

La búsqueda y selección del problema a resolver en el grupo experimental tuvo como consecuencia que los estudiantes desarrollaran ideas creativas más novedosas, originales y con una mayor elaboración que los que atendieron el problema contextualizado ya establecido de antemano por el facilitador en el grupo de control. Esto puede ser un indicador de que los participantes del grupo experimental estuvieron más motivados durante el proceso creativo con respecto a los del grupo de control, en concordancia con lo establecido por Amabile (1996) quien estableció que la creatividad depende críticamente de la motivación intrínseca y con Richardson y Mishra (2018), quienes demostraron que la libertad de elección del problema conduce a niveles más altos de motivación intrínseca y apoya la creatividad.

Con respecto a la dimensión elaboración, que tiene que ver con el grado en que la idea es completa, detallada y comprensible, el resultado obtenido fue significativamente mayor en el grupo experimental 
que en el grupo de control, evidenciando que los estudiantes que seleccionaron el problema tuvieron una mayor dedicación y compromiso con la creación de su idea, que los del grupo de control en el que el problema no fue elegido por ellos. Este resultado está en concordancia con lo establecido por FloresFuentes y Juárez-Ruiz (2017), quienes encontraron que cuando los estudiantes trabajan en proyectos resolviendo problemas contextualizados se propicia una mejora de sus actitudes.

Se emplearon en un proceso contextualizado las técnicas ficha de idea y sesión de creatividad con una modificación en una de sus etapas para promover la generación de ideas creativas y valorar su calidad. Se encontró evidencia de que el planteamiento de problemas es un predictor significativo de la calidad creativa de las ideas propuestas para solucionarlo. Los puntajes obtenidos en las dimensiones novedad, originalidad y elaboración de las ideas de los estudiantes que buscaron y plantearon el problema, respecto de los que atendieron el problema propuesto por el facilitador, confirman que el planteamiento de problemas puede fomentar el desarrollo de la creatividad, la dedicación y el compromiso con la actividad.

El trabajo también planteó una propuesta sobre cómo crear estrategias de trabajo y ambientes propicios para promover la generación de ideas creativas en estudiantes o trabajadores, mediante la búsqueda y planteamiento de problemas contextualizados. Donde se hace significativo el empleo de una técnica concreta que facilita el proceso de ideación y de valoración de calidad de la idea: la ficha de idea.

\section{Referencias}

Amabile, T. M. (1996). Creativity in context: update to "The social psychology of creativity". Westview Press.

Amabile, T. M. (2013). Componential theory of creativity. En E. H. Kessler (Ed.), Encyclopedia of Management Theory (Vol. 1, pp. 135-139). Sage. https://www.doi.org/10.4135/9781452276090.n50

Baer, J. y Kaufman, J. C. (2019). Assessing creativity with the consensual assessment technique. En I. Lebuda y V. P. Glăveanu (Eds.), The Palgrave handbook of social creativity research. Palgrave studies in creativity and culture (pp. 27-37). Palgrave Macmillan.

Barrera-Mora, F. y Reyes-Rodríguez, A. V. (2017). Tareas con diversas soluciones: estructura conceptual en profesores de matemáticas. Revista Electrónica de Investigación Educativa, 19(1), 110-122.

https://doi.org/10.24320/redie.2017.19.1.971

Benoit, G. (2011). Innovación. Grupo Editorial Patria.

Bretschneider, U., Rajagopalan, B. y Leimeister, J. M. (enero de 2012). Idea generation in virtual communities for innovation: The influence of participants' motivation on idea quality. Actas 45 Annual Hawaii International Conference on System Sciences (pp. 3467-3479).

https://doi.org/10.1109/HICSS.2012.13

Byrge, C. y Tang, C. (2015). Embodied creativity training: Effects on creative self-efficacy and creative production. Thinking Skills and Creativity, 16, 51-61. https://doi.org/10.1016/j.tsc.2015.01.002

Campbell, D. y Stanley, J. (1973). Diseños experimentales y cuasiexperimentales en la investigación social. Amorrortu.

Csikszentmihalyi, M. (2014). The systems model of creativity. Springer.

Elisondo, R. (2015). La creatividad como perspectiva educativa. Cinco ideas para pensar los contextos creativos de enseñanza y aprendizaje. Actualidades Investigativas en Educación, 15(3), 1-23. 
Flores-Fuentes, G. y Juárez-Ruiz, E. L. (2017). Aprendizaje basado en proyectos para el desarrollo de competencias matemáticas en Bachillerato. Revista Electrónica de Investigación Educativa, 19(3), 71-91. https://doi.org/10.24320/redie.2017.19.3.721

Galvao, A., Fleith, D. y Alencar, E. (2009). La relación entre creatividad y expertise: contribuciones teóricas y empíricas. Psicología, 27(1), 55-78. https://doi.org/10.18800/psico.200901.003

García, O., Pérez, R. y Miranda, A. (2018). Los profesores-investigadores universitarios y sus motivaciones para transferir conocimiento. Revista Electrónica de Investigación Educativa, 20(3), 43-55.

https://doi.org/10.24320/redie.2018.20.3.1754

Gold, R. (2007). The plenitude: Creativity, innovation, and making stuff. MIT Press.

Gruner, D. y Csikszentmihalyi, M. (2019). Engineering creativity in an age of artificial intelligence. En I. Lebuda y V. Glăveanu (Eds.), The Palgrave handbook of social creativity research. Palgrave studies in creativity and culture (pp. 447-462). Palgrave Macmillan.

Lapoujade, M. N. (1988). Filosofía de la imaginación. Siglo XXI.

Liao, Y. H., Chen, Y. L., Chen, H. C. y Chang, Y. L. (2018). Infusing creative pedagogy into an English as a foreign language classroom: Learning performance, creativity, and motivation. Thinking Skills and Creativity, 29, 213-223. https://doi.org/10.1016/j.tsc.2018.07.007

Newton, D. P. (2013). Moods, emotions and creative thinking: A framework for teaching. Thinking Skills and Creativity, 8, 34-44. https://doi.org/10.1016/i.tsc.2012.05.006

Ordaz, G., Acle, G. y Armenta, M. (2016). Crear con ciencia: programa de enriquecimiento para el desarrollo de la creatividad mediante experimentos científicos. En M. Valadez, G. López, M. Borges, J. Betancourt y R. Zambrano (Coords.), Programas de intervención para niños con altas capacidades y su evaluación (Cap. 7). Manual Moderno.

Perry, A., y Karpova, E. (2017). Efficacy of teaching creative thinking skills: A comparison of multiple creativity assessments. Thinking Skills and Creativity. https://doi.org/10.1016/i.tsc.2017.02.017

Plucker, J. A., Beghetto, R. A. y Dow, G. T. (2004). Why isn't creativity more important to educational psychologists? Potentials, pitfalls, and future directions in creativity research. Educational Psychologist, 39(2), 83-96. https://doi.org/10.1207/s15326985ep3902 1

Richardson, C. y Mishra, P. (2018). Learning environments that support student creativity: Developing the SCALE. Thinking Skills and Creativity, 27, 45-54. https://doi.org/10.1016/j.tsc.2017.11.004

Robinson, K. y Aronica, L. (2014). El elemento: descubrir tu pasión lo cambió todo. Vintage Español.

Tran, T. B. L., Ho, T. N., Mackenzie, S. V. y Le, L. K. (2017). Developing assessment criteria of a lesson for creativity to promote teaching for creativity. Thinking Skills and Creativity, 25, 10-26.

https://doi.org/10.1016/i.tsc.2017.05.006

Vally, Z., Salloum, L., AlQedra, D., El Shazly, S., Albloshi, M., Alsheraifi, S. y Alkaabi, A. (2019). Examining the effects of creativity training on creative production, creative self-efficacy, and neuro-executive functioning. Thinking Skills and Creativity, 31, 70-78. https://doi.org/10.1016/j.tsc.2018.11.003

Wu, M., Siswanto, I. y Mahfud, T. (2018). The role of cognitive and affective behavior in predicting the creative thinking of university students. International Journal of Innovation, Creativity and Change, 4(2), 90103. https://www.ijicc.net/images/vol4iss2/Mingchang Wu 2018.pdf 
Zatarain, R. (2018). Reconocimiento afectivo y gamificación aplicados al aprendizaje de Lógica algorítmica y programación. Revista Electrónica de Investigación Educativa, 20(3), 115-125.

https://doi.org/10.24320/redie.2018.20.3.1636 